\title{
Occult Pulsatile Tinnitus in Association with a Prominent Vein of Hypoglossal Canal: Two Case Reports
}

\author{
Baomin $\mathrm{LI}^{1}$, Xianli $\mathrm{LV}^{2}$, Zhongxue $\mathrm{WU}^{2}$, Xiangyu $\mathrm{CAO}^{1}$, Jun WANG ${ }^{1}$, Aili GE${ }^{1}$, Xinfeng $\mathrm{LIU}^{1}$, Sheng $\mathrm{LI}^{1}$ \\ ${ }^{1}$ General Hospital of PLA, Department of Neurosurgery, Beijing, China \\ ${ }^{2}$ Beijing Neurosurgical Institute and Beijing Tiantan Hospital, Capital Medical University, Beijing, China
}

\section{ABSTRACT}

The pathway of the vein of hypoglossal canal has not been mentioned in venous origin pulsatile tinnitus. We wished to clarify the possible complications related to this venous variant. We described 2 rare cases of troublesome pulsatile tinnitus associated with prominent vein of hypoglossal canal, which communicates with the jugular bulb and the marginal sinus. Both cases were successfully treated by positioning a stent across the vein of hypoglossal canal and jugular bulb. The 2 present clinical cases represent the first report of such a condition. The therapeutic decision-making is discussed in relation to the persistent pulsatile tinnitus and the etiopathologic hypothesis put forward.

KEYWORDS: Vein of hypoglossal canal, Jugular bulb, Pulsatile tinnitus

\section{INTRODUCTION}

$\mathrm{P}$ ulsatile tinnitus of vascular origin results from turbulent blood flow that is caused by increased flow volume or lumen irregularity $(4,9,19)$. Arterial causes of pulsatile tinnitus can be differentiated from venous causes by gently compressing the internal jugular vein ipsilateral to the tinnitus (7). This maneuver results in immediate resolution of tinnitus of venous origin but amplifies that of arterial origin. The venous causes of pulsatile tinnitus include diverticulum or aneurysm of the transverse-sigmoid sinus $(7,11,12,18)$, prominent or stenotic sigmoid sinus $(5,8,13,14,16)$, superficial lateral course of sigmoid sinus $(10,17)$, jugular bulb diverticulum $(5,14)$, high dehiscent jugular bulb (2), abnormal mastoid emissary vein and abnormal posterior condylar emissary vein $(3,6)$. Although pathologic causes for the symptoms are always given strong consideration, normal variations should also be included in a possible differential diagnosis. We describe 2 cases of pulsatile tinnitus associated with a vein of hypoglossal canal shown by cerebral angiography.

\section{CASE REPORTS}

Case 1

A 35-year-old woman, with no other medical history, presented with a 3-year history of a persistent right-sided pulsatile roaring tinnitus. The bruit interfered with her sleeping and daily function. Otoscopic and neurological examinations were normal. Compression of the right internal jugular vein produced subjective resolution of her symptoms. Magnetic resonance venogram (MRV) examination demonstrated a hypoplasia of the left transverse and sigmoid sinuses, prominent occipital sinus and vein of hypoglossal canal and jugular bulb. Digital subtraction angiography demonstrated the left dilated vein of hypoglossal canal (Figure 1A). The left transverse sigmoid sinus was hypoplastic.

Antiplatelet therapy consisting of orally administered aspirin $(100 \mathrm{mg})$ and clopidogrel $(75 \mathrm{mg})$ was initiated 3 days before the procedure. Endovascular procedure was performed under general anesthesia and systemic heparinization. During the procedure, heparin was given by intravenous infusion to maintain the activated clotting time at $>2$ times baseline, 
with a target of at least 300 seconds. Percutaneous access was gained through the femoral vein. Guiding catheters (8F) were advanced into the right internal jugular vein. Predilation with $4.0 \times 20 \mathrm{~mm}$ angioplasty balloon (Aviator, Cordis, USA) was performed at $8 \mathrm{~atm}$. A $4.5 \mathrm{~mm}-30 \mathrm{~mm}$ Wingspan stent (Boston Scientific, USA) was introduced into the hub of the Vasco catheter (Figures 1B, C). Under road-mapping, the stent was then pushed through the microcatheter and aligned directly across vein of hypoglossal canal. After treatment, postoperative examination showed no neurological deficits and immediate resolution of her symptoms. She was maintained on aspirin and clopidogrel for 1 month and aspirin (100 mg per day) for 6 months. There was no recurrence of the pulsatile tinnitus after 6 months.

\section{Case 2}

A 22-year-old woman had a 2-year history of progressive rightsided pulsatile tinnitus. The patient had been in otherwise good health with no similar previous episodes and no history of trauma. Physical examination revealed a normal tympanic membrane with no evidence of a vascular mass. No bruit was audible. No hearing deficit could be detected. Gentle pressure applied to the upper neck could stop the tinnitus. The pulsatile sound was not audible with a stethoscope. MRV showed an unusual drainage pathway of the torcular sinus, which drained into the occipital sinus, the marginal sinus and the vein of hypoglossal canal, and into the jugular bulb. Bilateral transverse sigmoid sinuses were hypoplastic. The cerebral venous drainage through the right vein of hypoglossal canal was excessively developed. Cerebral angiography confirmed these findings (Figure 2A).

A combination of $100 \mathrm{mg}$ of aspirin and $75 \mathrm{mg}$ of clopidogrel started 3 days before stent placement. The procedure was done under general anesthesia with monitoring by anesthesiologists. Transfemoral venous approach was performed and
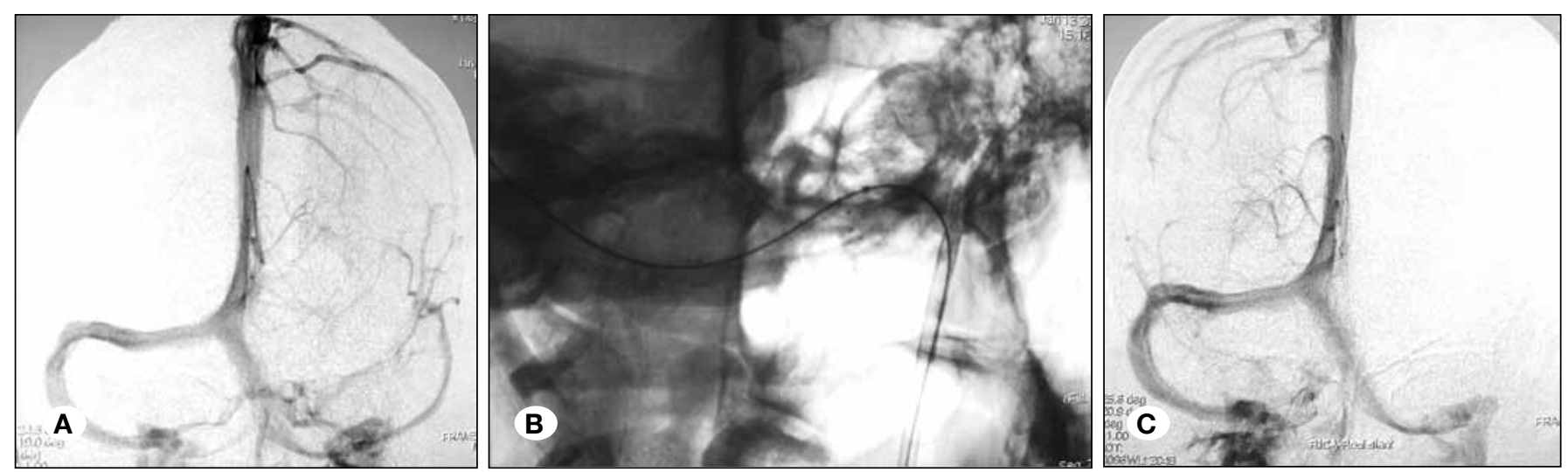

Figure 1: A) Sinus phase of the left carotid artery injection demonstrating a hypoplasia of the left transverse and sigmoid sinuses with a prominent drainage pathway of occipital sinus, the marginal sinus and the vein of hypoglossal canal, and into the jugular bulb. B) X-ray film showing the stent was deployed. C) Sinus phase of the right carotid artery injection after stent placement showing a more laminar blood flow through the hypoglossal canal vein.
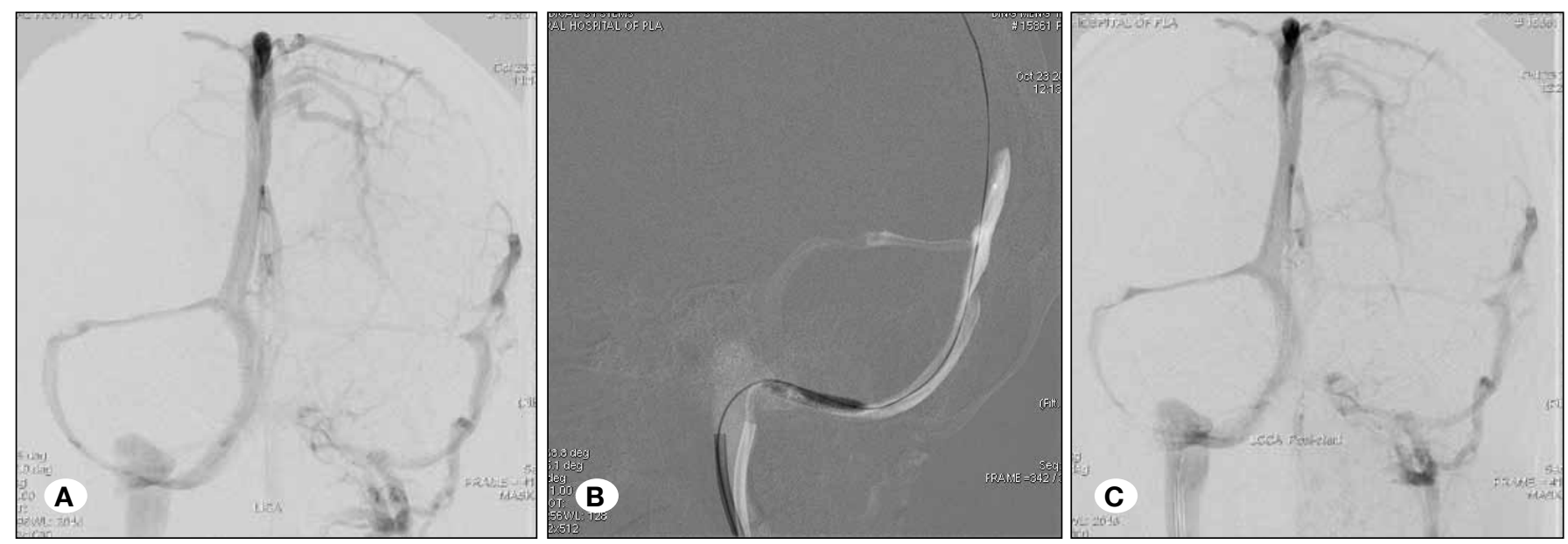

Figure 2: A) Sinus phase of the left carotid artery injection showing bilateral hypoplastic transverse and sigmoid sinuses. The cerebral venous drainage through the right occipital sinus, marginal sinus and vein of hypoglossal canal was excessively developed. B) Fluoroscopic image showing that a 4-mm $\times 20-\mathrm{mm}$ angioplasty balloon in the vein of hypoglossal canal was predilated. C) Sinus phase of the left carotid artery injection after deployment of a $6-\mathrm{mm} \times 30-\mathrm{mm}$ Solitaire stent showing more blood was shunted into the vein of hypoglossal canal. 
an $8 \mathrm{~F}$ guiding catheter was introduced into the internal jugular vein. A heparin bolus was given immediately before the interventional part of the procedure to increase the activated clotting time (ACT) to a minimum of 300 seconds. The vein of hypoglossal canal was predilated with a $4-\mathrm{mm} \times 20-\mathrm{mm}$ angioplasty balloon (Aviator, Cordis, USA) (Figure 2B). A 6-mm $\times 30-$ $\mathrm{mm}$ Solitaire stent (Covidien, USA) was deployed across the vein of hypoglossal canal (Figure 2C). She was maintained on aspirin and clopidogrel for 1 month and aspirin $(100 \mathrm{mg}$ per day) for 6 months. There was no recurrence of the pulsatile tinnitus after 2 months.

\section{DISCUSSION}

The development of cerebral venous drainage pathways has been well depicted in human anatomic studies. The pathway of the vein of hypoglossal canal, however, has not been mentioned in venous origin pulsatile tinnitus. We wished to clarify the possible complications related to this venous variant. The vein of hypoglossal canal is cranial emissary vein, which communicates with the jugular bulb and the marginal sinus (15). The diameter of the vein of hypoglossal canal is usually as small as that of a silk thread. It occasionally may be as large as 2 to $4 \mathrm{~mm}$, as shown by our series. The vein of hypoglossal canal can be so well developed that it can give a loud "venous" pulsatile bruit and a thrill.

One entity that has been described many times in the otolaryngologic literature but often forgotten is the essential type of tinnitus or cervical venous hum $(9,13)$. Essential tinnitus is nearly always pulse synchronous in nature because of the distinct pulsation in the intracranial venous system, as well as in the adjacent extracranial venous system (13). Essential tinnitus is thought to be most likely related to the distortion of laminar flow within the internal jugular vein after compression of the vein by the adjacent transverse process of the atlas and sternocleidomastoid muscle when the head is turned, usually away from the side of the tinnitus $(3,6,9)$. In the present case, the abnormal MRV findings and the lack of familiarity with the entity along with uncertainty as to whether this truly was the cause of the tinnitus prompted further investigation with cerebral angiography. The exact cause of the tinnitus and its pulsatile nature are difficult to understand. It is most likely related to turbulent blood flow within the tortuous venous sinus, as has been suggested in the case of a jugular bulb deformity $(2,5,14)$ and similar to the turbulent flow expected within the abnormal emissary vein in the case of venous hum $(3,6)$.

Therapeutic options in such a situation are limited. Occlusion of the sigmoid sinus or ligation of the jugular vein has been suggested if an expanding vascular mass has been excluded and adequate contralateral venous drainage is seen to be present $(3,6)$. In this case, MRV and cerebral angiography demonstrated no significant flow through the transverse and sigmoid sinuses. Therefore, stentplasty of the vein of hypoglossal canal was offered to the 2 patients. This technique based on our much experiences in stenting treatment of venous sinus stenosis causing pulsatile tinnitus $(1,8)$. We postulate that by changing flow characteristics through the hypoglossal canal emissary vein accounting for the beneficial result. We must assume that in some manner the flow became more laminar as more blood was shunted into the emissary vein.

\section{CONCLUSION}

Prominent vein of hypoglossal canal represents a rare cause of pulsatile tinnitus and can be treated by stent deployment. MRV and cerebral angiography can be helpful in excluding a vascular anomaly, such as venous variations.

\section{- REFERENCES}

1. Cao XY, Li BM, Li S: Intravascular stent therapy for cerebral venous sinus tinnitus. Zhonghua $\mathrm{Er}$ Bi Yan Hou Tou Jing Wai Ke Za Zhi 45(9):776, 2010 (in Chinese)

2. El-Begermy MA, Rabie AN: A novel surgical technique for management of tinnitus due to high dehiscent jugular bulb. Otolaryngol Head Neck Surg 142(4):576-581, 2010

3. Forte V, Turner A, Liu P: Objective tinnitus associated with abnormal mastoid emissary vein. J Otolaryngol 18(5): 232-235, 1989

4. Hofmann E, Nadjmi M, Ratzka M, Schuknecht B: Pulse synchronous vascular tinnitus: Radiologic diagnosis and therapy. HNO 35(5):211-218, 1987 (in German)

5. Horne SK, Lowry TR, Horlbeck DM: Radiology quiz case 2. Hypoplasia of the left transverse dural sinus and a prominent right transverse sinus and jugular bulb causing rightsided objective tinnitus. Arch Otolaryngol Head Neck Surg 130(6):797, 799-800, 2004

6. Lambert PR, Cantrell RW: Objective tinnitus in association with an abnormal posterior condylar emissary vein. Am J Otol 7(3):204-207, 1986

7. Lenck S, Mosimann PJ, Labeyrie MA, Houdart E: Pulsatile tinnitus caused by an aneurysm of the transverse-sigmoid sinus: A new case report and review of literature. J Neuroradiol 39(4):276-279, 2012

8. Li BM, Cao XY, Wang QJ, Wang J, Ge AL, Liu XF, Li Q, Li $\mathrm{S}$ : Stenting treatment of venous sinus stenosis for intractable pulsatile tinnitus. Zhonghua Yi Xue Za Zhi 92(17):1179-1182, 2012 (in Chinese)

9. Lv X, Jiang C, Li Y, Liu L, Liu J, Wu Z: Transverse-sigmoid sinus dural arteriovenous fistulae. World Neurosurg 74:297305, 2010

10. Mehall CJ, Wilner HI, LaRouere MJ: Pulsatile tinnitus associated with a laterally placed sigmoid sinus. AJNR Am J Neuroradiol 16 Suppl 4:905-907, 1995

11. Mehanna R, Shaltoni $H$, Morsi $H$, Mawad M: Endovascular treatment of sigmoid sinus aneurysm presenting as devastating pulsatile tinnitus. A case report and review of literature. Interv Neuroradiol 16(4):451-454, 2010

12. Park YH, Kwon HJ: Awake embolization of sigmoid sinus diverticulum causing pulsatile tinnitus: Simultaneous confirmative diagnosis and treatment. Interv Neuroradiol 17(3):376-379, 2011 
13. Russell EJ, De Michaelis BJ, Wiet R, Meyer J: Objective pulse-synchronous "Essential" tinnitus due to narrowing of the transverse dural venous sinus. Int Tinnitus J 1(2):127-137, 1995

14. Signorelli F, Mahla K, Turjman F: Endovascular treatment of two concomitant causes of pulsatile tinnitus: Sigmoid sinus stenosis and ipsilateral jugular bulb diverticulum. Case report and literature review. Acta Neurochir (Wien) 154(1):89-92, 2012

15. Tubbs RS, Ammar K, Liechty P, Wellons JC 3rd, Blount JP, Salter EG, Oakes WJ: The marginal sinus. J Neurosurg 104(3): 429-431, 2006
16. Wee JH, Song JJ, Koo JW, Kim CS: Increased intracranial pressure after surgical treatment of pulsatile tinnitus from a prominent sigmoid sinus. Otol Neurotol 33(6):e41-42, 2012

17. Xue J, Li T, Sun X, Liu Y: Focal defect of mastoid bone shell in the region of the transverse-sigmoid junction: A new cause of pulsatile tinnitus. J Laryngol Otol 126(4):409-413, 2012

18. Zenteno M, Murillo-Bonilla L, Martínez S, Arauz A, Pane C, Lee $A$, del Valle R: Endovascular treatment of a transversesigmoid sinus aneurysm presenting as pulsatile tinnitus. Case report. J Neurosurg 100(1):120-122, 2004

19. Zhang Y, Wang W, Dai C, Chen L: Diagnosis and management of pulsatile tinnitus of venous origin. Lin Chung $\mathrm{Er} \mathrm{Bi}$ Yan Hou Tou Jing Wai Ke Za Zhi 24(6):267-269, 2010 (in Chinese) 